\title{
Arginase-1 Peptide Vaccine
}

National Cancer Institute

\section{Source}

National Cancer Institute. Arginase-1 Peptide Vaccine. NCI Thesaurus. Code C161833.

A vaccine comprised of arginase-1 peptides, with potential antineoplastic activity. Upon vaccination, the arginase-1 peptide vaccine may activate the immune system to induce an immune response against arginase-1-expressing cells. Arginase-1 is expressed by some cancer cells and by immune inhibitory cells, such as myeloid-derived suppressor cells (MDSCs) and tumor-associated macrophages (TAMs); its expression is associated with poor prognosis. 